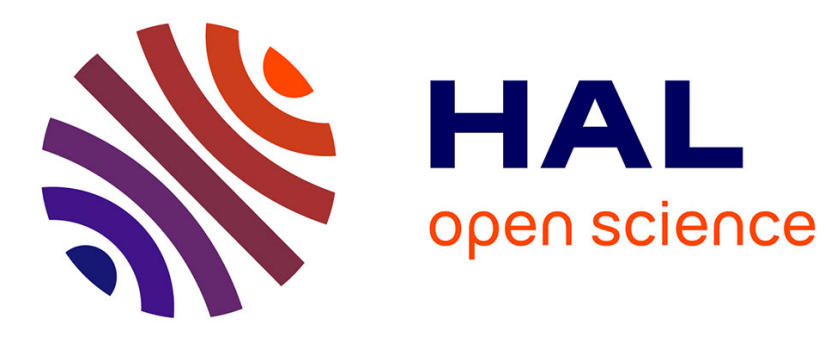

\title{
Benchmarking DFT surface energies with Quantum Monte Carlo
}

\author{
Simon John Binnie, Ester Sola, Dario Alfè, Michael J Gillan
}

\section{To cite this version:}

Simon John Binnie, Ester Sola, Dario Alfè, Michael J Gillan. Benchmarking DFT surface energies with Quantum Monte Carlo. Molecular Simulation, 2009, 35 (07), pp.609-612. 10.1080/08927020802635145 . hal-00515070

\section{HAL Id: hal-00515070 \\ https://hal.science/hal-00515070}

Submitted on 4 Sep 2010

HAL is a multi-disciplinary open access archive for the deposit and dissemination of scientific research documents, whether they are published or not. The documents may come from teaching and research institutions in France or abroad, or from public or private research centers.
L'archive ouverte pluridisciplinaire HAL, est destinée au dépôt et à la diffusion de documents scientifiques de niveau recherche, publiés ou non, émanant des établissements d'enseignement et de recherche français ou étrangers, des laboratoires publics ou privés. 


\section{Molecular Simulation \\ Journal of \\ Experimental Nanoscience

\section{Benchmarking DFT surface energies with Quantum Monte Carlo}

\begin{tabular}{|c|c|}
\hline Journal: & Molecular Simulation/Journal of Experimental Nanoscience \\
\hline Manuscript ID: & GMOS-2008-0215.R1 \\
\hline Journal: & Molecular Simulation \\
\hline $\begin{array}{r}\text { Date Submitted by the } \\
\text { Author: }\end{array}$ & 14-Nov-2008 \\
\hline Complete List of Authors: & $\begin{array}{l}\text { Binnie, Simon; University College London, Physics \& Astronomy } \\
\text { Sola, Ester; University College London, Earth Sciences; University } \\
\text { College London, Materials Simulation Laboratory } \\
\text { Alfè, Dario; University College London, Earth Sciences; University } \\
\text { College London, Materials Simulation Laboratory; University College } \\
\text { London, Physics \& Astronomy; University College London, London } \\
\text { Centre for Nanotechnology } \\
\text { Gillan, Michael; University College London, Materials Simulation } \\
\text { Laboratory; University College London, Physics \& Astronomy; } \\
\text { University College London, London Centre for Nanotechnology }\end{array}$ \\
\hline Keywords: & DFT, surface-energies, DMC, $\mathrm{LiH}, \mathrm{MgO}$ \\
\hline
\end{tabular}

\section{SCHOLARONE Manuscripts}




\title{
PRELIMINARY COMMUNICATIONS
}

\section{Benchmarking DFT surface energies with quantum Monte Carlo}

\author{
S.J. Binnie ${ }^{a, c, d *}$, E. Sola ${ }^{a, b}$, D. Alfè $\grave{a, b, c}^{a, d}$ and M.J. Gillan ${ }^{a, c, d}$ \\ ${ }^{a}$ Materials Simulation Laboratory, University College London, London, UK; ${ }^{b}$ Dept. \\ of Earth Sciences, University College London, London, UK; ${ }^{c}$ Dept. of Physics and \\ Astronomy, University College London, London, UK; ${ }^{d}$ London Centre for Nanotechnology, \\ University College London, London, UK
}

\begin{abstract}
Previous work has demonstrated that for many materials, surface energies $\sigma$ calculated by Density Functional Theory (DFT) methods depend significantly on the exchange-correlation (xc) functional used. This could pose significant problems when using DFT for predicting structures of nanocrystals both in vacuum and on substrates. Here we present initial results from a systematic study of $\sigma$, for a series of ionic materials using the VASP code and its projector augmented wave implementation of DFT. Calculations on $\mathrm{LiH}$ and $\mathrm{MgO}$ presented here were performed using four of the functionals available in VASP (LDA, PW91, PBE and $\mathrm{RPBE}$ ) along with the recent $\mathrm{Wu}-\mathrm{Cohen}$ modification of PBE. The results we present show that there is indeed a significant variation in $\sigma$ due to differing functionals. Furthermore we are able to ascertain which functionals gave the most accurate results by performing calculations of $\sigma$ for $\mathrm{LiH}$ using diffusion quantum Monte Carlo methods which are generally accepted to be significantly more accurate, albeit more expensive, in calculating these quantities.
\end{abstract}

Keywords: DFT, DMC, surface-energy, $\mathrm{LiH}, \mathrm{MgO}$.

\section{Introduction}

Over the past twenty years Density Functional Theory (DFT) $[1,2]$ has become the most popular electronic structure method for a variety of applications. This is mainly due to its relative low-cost and general accuracy for the systems being studied. The main approximation in DFT is, of course, the form of the exchangecorrelation functional (xc), having developed from the relatively naive local density approximation (LDA) through the various implementations of the generalized gradient approximation (GGA) to the more recent hybrid-functionals which include empirical data. Despite DFT's wide use in surface modelling there is evidence scattered throughout the literature that the results may be strongly affected by the xc used. When one considers the surface formation energy $(\sigma)$ which is the reversible work done per unit area in creating a surface in a given crystal the results are particularly worrying. Here the rather limited work available seems to suggest that this rather important quantity in surface science is consistently underestimated by about $30 \%$ by the more advance GGA xcs, whereas the more naive LDA approximation seems to reproduce experiment $[3,4]$.

In this study, from which were are presenting some preliminary results, we are looking at a variety of ionic solids. We firstly calculate the bulk properties of each solid using DFT and four different xcs, namely: the Perdew-Wang xc (PW91)[5], PBE[6],

\footnotetext{
*Corresponding author. Email: s.binnie@ucl.ac.uk.uk
} 
the revised form of PBE (rPBE) [7] and the recent Wu-Cohen modification of PBE (WC) [8]. From this we are able to see the expected dependence on $\mathrm{xc}$ of the various bulk properties. We then calculate the surface formation energy using the different functionals. As experimental data is simply unavailable for many systems and for others have error bars comparable to the differences between xcs we follow the previous work on this subject in using the Diffusion Monte Carlo (DMC) [9] method to produce a benchmark by which to measure the DFT functionals. DMC is a theoretically exact method where the wavefunction for the many-body Schrödinger equation is represented by a number of walkers, whose motion is governed by a drift-diffusion Green's function, whose evolution in imaginary time projects out the groundstate wavefunction. As this method models the same regime as DFT (zero temperature with no zero-point motion) and the only uncontrolled error is the 'fixed-node' error introduced by the fermion-sign problem this should provide a very good way of benchmarking the accuracy of the DFT values.

\section{Methods}

In order to reduce what would amount to a bias towards one particular method it was decided that the lattice parameter used for each xc and for QMC would be the equilibrium lattice parameter for that particular method, breaking from previous work where the experimental lattice parameter was used throughout. Hence the first step in the calculations was to obtain the bulk properties of the system for each method. This was done in the usual way by obtaining the total energy of the crystal at several different primitive cell volumes and then fitting this to a Birch-Murnaghan equation of state. When calculating the surface energies, previous studies have shown that the traditional method of just subtracting the appropriate amount of bulk crystal energy from the total energy of a given thickness of slab can lead to inaccurate results. We therefore decided to use a more recent method[10] where the total energy of several different thicknesses of slab are fitted to:

$$
E_{\text {slab }}=2 A \sigma+N E_{\text {bulk }}
$$

where $N$ is the number of layers, $A$ the surface area of the slab and $E_{b u l k}$ is the energy of the bulk crystal per layer (obtained here by fitting). This method allows for a significant cancellation of errors and as such seems to give good values for $\sigma$. There is also the question of the geometry of the atoms at the surface of the slab. With DFT it is fairly easy to let these atoms relax whereas with DMC this would be a particularly expensive and time consuming task (far more expensive in fact than the rest of the calculations.) One solution would have been to use the relaxed geometry from a DFT run in the DMC calculations but this would inherently bias the DMC result. Therefore it was decided that the DMC calculations would be done just with the bulk terminated slabs and the DFT calculations done with both bulk terminated and relaxed slabs. This would allow us to compare the DFT and DMC results directly, but also see how big an effect the surface relaxation actually is.

\subsection{DFT}

The DFT work consisted of projector augmented wave calculations done with the VASP[11] code and the pseudopotentials supplied with the code. The convergence of total-energy with respect to k-point sampling was achieved to within $1 \mathrm{meV}$ 
per formula unit. This required a $7 \times 7 \times 7$ Monkhorst-Pack grid for bulk $\mathrm{LiH}$ and $8 \times 8 \times 8$ for bulk $\mathrm{MgO}$. For the slabs there was also the thickness of the vacuum layer to be taken into account (as the simulation was periodic in the z-direction.) Here convergence of total-energy to within $10 \mathrm{meV}$ per layer was achieved with vacuum layers of $2 a_{0}$ for $\mathrm{LiH}$ and $\frac{3}{2} a_{0}$ for $\mathrm{MgO}$ (with $a_{0}$ being the equilibrium lattice paramater). Slabs of thickness between one and fifteen atomic layers were calculated with finite-size effects causing us to discard layers 1-2. In the end we achieved convergence for the surface energies of $0.002 \mathrm{~J} / \mathrm{m}^{2}$.

\section{2. $Q M C$}

The diffusion Monte Carlo method allows us to project out the exact groundstate for a given system. However due to the fermion-sign problem, for fermionic systems we are required to know a priori all the locations where the wavefunction is zero (the nodal surface.) The usual method therefore is to generate a trial wavefunction (in the form of a Slater determinant) using DFT which fixes the nodal surface. This is then multiplied by a Jastrow factor (containing usually electron-electron, electron-nucleus and electron-electron-nucleus correlation terms) determined by a variational Monte Carlo (VMC) calculation, in order to reduce the cost of the subsequent DMC calculations. This makes the method variational as we are only projecting out the lowest energy state for the given nodal-surface, however this error is usually quite small.

Thus we used the PWscf code from the Quantum Espresso[12] package to generate the trial wavefunction and the CASINO[13] code to perform the VMC and DMC calculations. In order to reduce the expense of the calculations the Dirac-Fock pseudopotentials by Trail \& Needs[14, 15] were used. These are quite hard and thus required quite a high plane-wave cut-off (300 Ry) in PWscf. The wavefunction was then re-expanded in b-spline functions (blips)[16] in order to increase the efficiency of the QMC calculations.

DMC is susceptible to two further errors, firstly the Green's function is only valid in the limit of an infinitely small timestep in the evolution in imaginary time. Convergence of the total energy to less than $10 \mathrm{meV}$ per formula unit was achieved with timesteps of 0.025 a.u. for the bulk and 0.01 a.u. for the slabs. Secondly DMC suffers from finite-size errors which come about due to the periodic interactions between simulation cells. These decrease as the size of the simulation cell is increased and can be corrected for. In the bulk crystal we used the recently proposed method of Kwee et al.[17] (implemented in PWscf by Dr. Ester Sola) which corrects for both the single-body and two-body effects. This involves correcting as follows:

$$
E=E_{D M C}(L)+E_{L D A}(\infty)-E_{K Z K}(L)
$$

where $E_{D M C}$ is the raw DMC energy at cell size $L \times L \times L, E_{L D A}(\infty)$ is the converged LDA energy and $E_{K Z K}(L)$ is the calculation done with the Kwee et al. xc at a single k-point and $E$ is the corrected total energy.

For the surface the KZK functional form breaks down due to its dependence on simulation cell volume so the usual single-body LDA correction was used:

$$
E=E_{D M C}(L)+E_{L D A}(\infty)-E_{L D A}(L)
$$

where $E_{L D A}(L)$ is the LDA energy at a single k-point.

Slabs of thickness between 1-6 atomic layers were calculated, with a free boundary condition on the z-axis. Slabs with one and two layers were neglected because of 
finite size effects.

\section{Results}

\section{Discussion}

As expected we have quite clearly seen a bias in the bulk parameters for the systems studied with respect to the xc used. Given the nature of the DFT simulations (zero-temperature and the lack of zero-point energy corrections) comparison with experiment here is not particularly useful, but the DMC values would suggest that the predictions of PW91 and PBE are good for LiH.

When looking at the surface formation energy this dependence is even more pronounced with $\mathrm{rPBE}$ producing energies of almost half that of the LDA. Clearly this is an unacceptably large variation. For $\mathrm{LiH}$ we can see that the PW91 and PBE xcs provide quite good agreement with DMC. However, previous results on both $\mathrm{Pb}$ surfaces[4] and $\mathrm{MgO}[3]$ have demonstrated good agreement between LDA and DMC, with the GGA functionals in these cases underestimating the DMC value by around $30 \%$. One explanation for this could be that DFT relies on lucky cancellations of errors in order to reproduce accurate surface energies. It is also good to note that the effect of relaxation of the surface structure on $\sigma$ is indeed small as we assumed, validating our approach of only comparing bulk-terminated surfaces with DMC.

We note that the previous DMC and DFT calculations on $\mathrm{MgO}[3]$ were performed with the experimental lattice parameter and as such more work needs to be done on the dependence of $\sigma$ on the lattice parameter for a fuller comparison to be made. Calculations are on-going to extend the number of systems studied and the DFT results seem to support the trends outlined here. An interesting possibility is the use of high-level quantum chemistry, which has already been successfully used to calculate the cohesive energy of $\mathrm{LiH}[18]$ and could also be used to calculate surface energies. This would be a great step forward and, of course, would give another independent benchmark with which to compare DFT and indeed DMC.

In summary: We have demonstrated that there is indeed a significant dependence of both bulk properties and surface energies on the DFT xc functional used and demonstrated, in the cases of $\mathrm{LiH}$ and $\mathrm{MgO}$, that relaxation of the surface contributes a relatively small amount to the total surface energy. Finally we have presented Diffusion Monte Carlo results for LiH showing the xcs that give the best agreement are the PW91 and PBE implementation of the GGA.

\section{References}

[1]P. Hohenberg and W. Kohn, Inhomogeneous electron gas, Phys. Rev. 136, B864-B871 (1964),

[2]W. Kohn and L. Sham, Self-Consistent Equations Including Exchange and Correlation Effects, Phys. Rev. 140, A1133-A1138 (1965),

[3]D. Alfè and M.J. Gillan, The energetics of oxide surfaces by quantum Monte Carlo, J. Phys.: Condens. Matter 18 (35), L435-L440 (2006),

[4]D. Yu and M. Scheffler, First-principles study of low-index surfaces of lead, Phys. Rev. B 70, 155417 $(2004)$

[5]J.P. Perdew, J.A. Chevary, S.H. Vosko et al., Atoms, molecules, solids, and surfaces: Applications of the generalized gradient approximation for exchange and correlation, Phys. Rev. B 46, 6671-6687 (1992),

[6]J.P. Perdew, K. Burke and M. Ernzerhof, Generalized gradient approximation made simple, Phys. Rev. Lett. 77, 3865-3868 (1996),

[7]B. Hammer, L.B. Hansen and J.K. Nørskov, Improved adsorption energetics within density-functional theory using revised Perdew-Burke-Ernzerhof functionals, Phys. Rev. B 59, 7413-7421 (1999),

[8]Z. Wu and R.E. Cohen, More accurate generalized gradient approximation for solids, Phys. Rev. B 73, $235116(2006)$, 
[9]W. Foulkes, L. Mitás, R. Needs and G. Rajagopal, Quantum Monte Carlo simulations of solids, Rev. Mod. Phys. 73, 33-83 (2001),

[10]V. Fiorentini and M. Methfessel, Extracting convergent surface energies from slab calculations, J. Phys.: Condens. Matter 8, 6525-6529 (1996),

[11] G. Kresse and J. Furthmüller, Efficient iterative schemes for ab initio total-energy calculations using a plane-wave basis set, Phys. Rev. B 54, 11169-11186 (1996),

[12]S. Baroni, A.D. Corso, S. de Gironcoli et al. . <http://www.pwscf.org >.

[13]R.J. Needs, M.D. Towler, N.D. Drummond and P. López Ríos, CASINO version 2.2 User Manual, University of Cambridge, Cambridge 2008.

[14]J.R. Trail and R.J. Needs, Smooth relativistic Hartree-Fock pseudopotentials for $H$ to Ba and Lu to Hg, J. Chem. Phys. 122 (17), 174109 (2005),

[15]_ Norm-conserving Hartree-Fock pseudopotentials and their asymptotic behavior, J. Chem. Phys. $122(1), 014112$ (2005),

[16]D. Alfè and M.J. Gillan, Efficient localized basis set for quantum Monte Carlo calculations on condensed matter, Phys. Rev. B 70, 161101 (2004),

[17]H. Kwee, S. Zhang and H. Krakauer, Finite-size correction in many-body electronic structure calculations, Phys. Rev. Lett. 100 (12), 126404 (2008),

[18]F.R. Manby, D. Alfè and M.J. Gillan, Extension of molecular electronic structure methods to the solid state: Computation of the cohesive energy of Lithium Hydride, Phys. Chem. Chem. Phys. 8, 5178-5180 (2006), 
Table 1. DFT bulk parameters: Lattice constant $\left(a_{0}\right)$ and bulk modulus $\left(B_{0}\right)$

Table 2. DFT surface energies

$$
\sigma\left(\mathrm{J} / \mathrm{m}^{2}\right)
$$

\begin{tabular}{ccccc}
\hline \multicolumn{5}{c}{$\sigma\left(\mathrm{J} / \mathrm{m}^{2}\right)$} \\
Functional & LiH & & \multicolumn{2}{c}{ MgO } \\
& Bulk-terminated & Relaxed & Bulk-terminated & Relaxed \\
\hline LDA & 0.4663 & 0.4586 & 1.1807 & 1.1662 \\
PW91 & 0.3435 & 0.3342 & 0.9233 & 0.9051 \\
PBE & 0.3365 & 0.3273 & 0.9107 & 0.8920 \\
RPBE & 0.2723 & 0.2598 & 0.7807 & 0.7592 \\
Wu-Cohen & 0.4105 & 0.4024 & 1.0276 & 1.0100 \\
\hline \multirow{2}{*}{ DMC } & $0.36(1)$ & - & - & - \\
\hline
\end{tabular}

